\title{
BRCA1 1675delA and 1135insA Account for One Third of Norwegian Familial Breast-Ovarian Cancer and Are Associated with Later Disease Onset than Less Frequent Mutations
}

\author{
Åke Borg ${ }^{1, \#}$, Anne Dørum ${ }^{2}$, Ketil Heimdal ${ }^{2}$, \\ Lovise Mæhle ${ }^{2}$, Eivind Hovig ${ }^{3}$, Pål Møller ${ }^{2}$ \\ ${ }^{\prime}$ Department of Oncology, University \\ Hospital, SE-221 85 Lund, Sweden \\ ${ }^{2}$ Unit of Medical Genetics, The Norwegian \\ Radium Hospital, N-0310 Oslo, Norway \\ ${ }^{3}$ Department of Tumour Biology, The \\ Norwegian Radium Hospital, N-0310 Oslo, \\ Norway
}

\begin{abstract}
A total of 845 women from breastovarian cancer kindreds were enrolled in a clinical follow-up program for early disease diagnosis; 35 women were prospectively identified with cancer. In order to estimate the role of genetic factors for cancer predisposition in this well-defined set of patients, considered as representative for familial breastovarian cancer in the Norwegian population, the BRCA1 gene was investigated for germline mutations. The entire coding region of BRCA1 was analysed using a protein truncation test, direct sequencing and a screen for known large genomic deletions and insertions. Twenty one $(60 \%)$ of the 35 patients were identified as carriers of 11 distinct BRCA1 mutations. Two previously described founder mutations, 1675delA and 1135insA, were found to account for more than half $(11 / 21)$ of all BRCA1 cases and for almost one third (11/35) of all breast and ovarian cancers. Supported by a previous population-based analysis of these founder mutations in ovarian cancer, our findings suggest that a significant proportion of women at risk for developing inherited breast and ovarian cancer can be identified. This is particularly

\footnotetext{
\# Correspondence: Pål Møller, Unit of Medical Genetics, The Norwegian Radium Hospital, N-0310 Oslo, Norway, Tel.: + 47229 35675, Fax: + 47229 35219, E-mail: pmoller@ulrik.uio.no
}

obvious in certain geographic regions where these founder mutations are prevalent. Women carrying the two founder mutations had a significantly older age of disease onset as compared to women with other BRCA1 mutations. This observation indicates that BRCA mutation penetrance estimates from populations with strong founder effects may be biased. One reason why some deleterious mutations are allowed to prevail in a population may be coupled to penetrance and the fact that they seldom induce disease in women in child-bearing ages. Eleven out of $12(92 \%)$ breast cancers in BRCA1 mutation carriers were estrogen receptor negative, versus 4 out of 9 $(44 \%)$ in mutation negative patients $(\mathrm{p}=0.03)$. Histopathological characteristics of the prospectively detected cancers indicated an unfavourable prognosis in mutation carriers.

\section{INTRODUCTION}

It has been estimated that $80 \%$ of all breastovarian cancer kindreds have BRCA1 mutations [7]. In Norway, familial breast-ovarian cancer kindreds are frequently seen, and we have previously reported that this may be due to the presence of BRCA1 founder mutations [1,3]. These founder mutations are probably related to the population bottleneck during the Bubonic plagues 25 generations ago, followed by a rapid population expansion.

We have previously found that $3 \%$ of Norwegian ovarian cancer patients carry BRCA1 $1675 \mathrm{delA}$ or 1135insA [4]. In the present study we analyzed women from breast-ovarian cancer kindreds, in whom breast and/or ovarian cancer were prospectively detected in our health care program. Our results confirm the significance of 
BRCA1 mutations in familial breast-ovarian cancer, and reinforce our assumptions of a high prevalence of two BRCA1 founder mutations in the Norwegian population. The results will allow us to estimate the frequency of BRCA1 induced breast and ovarian cancer in Norway, and will shed light on previous conflicting reports on the penetrance of BRCA mutations in different etnic groups.

\section{MATERIAL AND METHODS}

\section{Patients}

The study included 845 referred or selfreferred women from breast-ovarian cancer families that, based on their family history of cancer and without prior access to genetic testing, were offered annual clinical/mammographic screening for early disease diagnosis. Information is now available from more than one thousand breast or breast-ovarian cancer families, which may be regarded as representative for the Norwegian population. The selection criteria and clinical findings of prospective cancers in breast and breast-ovarian cancer kindreds have been reported previously. Breast cancer continued to occur in both breast and breast-ovarian cancer kindreds. We have estimated that $87 \%$ of prospectively demonstrated cancers may be inherited [10]. Ovarian cancer continued to occur in families with one woman with both breast and ovarian cancer, and/or in families including two or more ovarian cancers [5]. In the present study, the women with prospective cancers in breast-ovarian cancer kindreds were subjected to BRCA1 mutation analyses. The series comprised thirty-five women from 30 independent families diagnosed with 23 breast tumours and 16 ovarian cancers during the period from June 1992 to June 1997. Twenty-six of these 35 women had no sign or symptom of cancer at inclusion, whereas seven patients with prospectively diagnosed ovarian cancer had previously experienced breast cancer. Two patients with prospectively diagnosed breast cancer, had previously experienced ovarian cancer. Further details on age of onset are given in Table 1. We considered these 35 women as representative of Norwegian breast-ovarian cancer kindreds and decided to determine BRCA1 mutations to arrive at prevalence estimates.

\section{Mutation analysis}

Genomic DNA from peripheral blood was used for PCR-based analysis of the BRCA1 gene, basically as previously described [8]. The large exon 11 was subdivided into three overlapping PCR-fragments and screened for mutations causing premature translation termination using a protein truncating test (PTT). The remaining 21 coding exons and the 5' end of exon 11 (not covered by PTT) were analyzed by direct sequencing using an $\mathrm{ABI} 373 \mathrm{~A}$ or $\mathrm{ABI} 310$ instrument and ET-Dyenamics (Amersham Inc) or BigDye terminator (Perkin-Elmer) chemistry. The two founder mutations, 1135insA and 1675delA, were specifically identified by multiplex PCR followed by gel electrophoresis permitting detection of single basepair length variations, followed by sequencing on an Alf Express ${ }^{\mathrm{TM}}$ (Pharmacia Biotech) [4]. The following previously described genomic deletions or insertions were analysed using specifically designed primers and positive control DNA (provided by Peter Devilee, University of Leiden, and Sylvie Mazoyer, IARC, Lyon): exons 13-16/14kb deletion, exon 13/3.8kb deletion, exon $22 / 510 \mathrm{bp}$ deletion, exon 13/6kb duplication.

\section{Histopathological examination}

All tumours were histopathologically examined and classified according to FIGO stage and pTNM criteria.

\section{Statistical analysis}

Associations were sought for with Fisher's exact $p$ (one-sided), group differences with respect to age of onset were considered by Mann-Whitney U-tests (one-sided).

RESULTS 
Results from mutation analyses, classification of observed cancers and ages at diagnoses are given in Table 1. Breast cancer was found in 20 patients, including 22 infiltrating tumours (three of which were bilateral) and one in situ carcinoma. Ovarian cancer was found in 16 patients, and included 11 infiltrating epithelial cancers, four borderline ovarian cancers and one granulosa cell tumour. One patient was prospectively diagnosed with both a borderline ovarian cancer and a breast carcinoma.

Table 1

Age at prospective cancers diagnosed, type of cancer, and BRCA1 mutation. $\mathrm{DC}=$ ductal cancer, gr $=$ grade, $\mathrm{st}=$ stage, $\mathrm{ER}=$ oestrogen receptor, $\mathrm{PgR}=$ progesterone receptor, $\mathrm{BL}=$ borderline. Results not given were not examined for

\begin{tabular}{|c|c|c|c|c|c|c|}
\hline Fam-Pid & BRCA1 mut & Breast tumour characteristics & $\begin{array}{l}\text { Age } \\
\text { breast } \\
\text { cancer }\end{array}$ & Ovarian tumour characteristics & $\begin{array}{l}\text { Age } \\
\text { ovarian } \\
\text { cancer }\end{array}$ & $\begin{array}{l}\text { Age } \\
\text { previous } \\
\text { cancer }\end{array}$ \\
\hline $32-22$ & & DC, T1N0M0, ER-/PgR- & 53 & & & \\
\hline $32-43$ & & DC in situ & 41 & & & \\
\hline $71-9$ & 913delCT & & & Serous, grIII, stIII & 35 & \\
\hline $128-3$ & & & & BL mucinous cystadenoma, stI & 54 & BC $42 / 51$ \\
\hline $155-3$ & & & & BL serous cystadenoma, stI & 53 & BC 46 \\
\hline $162-1$ & 1675delA & DC, grIII, T1N0M0, ER-/PgR- & 61 & & & \\
\hline $162-2$ & 1675 delA & & & Serous, grIII, stIII & 65 & \\
\hline $180-5$ & 1135insA & DC, grIII, T1N1M0, ER-PgR- & 58 & BL serous cystadenoma, stI & 58 & \\
\hline $180-6$ & 1135insA & DC, grIII, T2NOM0, ER-/PgR- & 37 & & & \\
\hline $208-2$ & & DC, grII, T1N0M0 & 37 & & & \\
\hline $220-1$ & & & & BL serous cystadenoma, stI & 44 & \\
\hline $253-9$ & C2988T & & & Serous/endometroid, grIII, stIII & 51 & BC 48 \\
\hline $266-2$ & & DC, grI, T1N1M0, ER+/PgR+ & 42 & & & \\
\hline $302-1$ & 4148delTCAA & DC, T1N0M0, ER-/PgR- & 35 & & & OC 34 \\
\hline $315-1$ & $\mathrm{xxxx}^{*}$ & DC, grIII, T1N0M0, ER-/PgR- & 43 & & & \\
\hline $316-1$ & 1675delA & & & Adenocarcinoma, grIII, stII & 48 & BC $36 / 40$ \\
\hline $316-2$ & 1675delA & & & Serous/endometroid, grIII, stIII & 50 & BC 37 \\
\hline $373-3$ & G5166T & & & Serous, grIII, stIII & 41 & \\
\hline $381-3$ & & DC, grII, T1N0M0, ER+/PgR+ & 38 & & & \\
\hline $397-2$ & 1135 ins A & DC, grII, T2N0M0, ER-/PgR+ & 48 & & & \\
\hline $437-6$ & 816delGT & $\begin{array}{l}\text { DC, grIII, T2N0M0, ER-/PgR- } \\
\text { DC, grIII, T2N0M0, ER-/PgR- }\end{array}$ & $\begin{array}{l}34 \\
36\end{array}$ & & & \\
\hline $601-10$ & $1675 \mathrm{delA}$ & & & Serous/endometroid, grIII, stIII & 44 & \\
\hline $603-3$ & & $\begin{array}{l}\text { DC, T1N0M0, ER+/PgR+ } \\
\text { DC, grI, T1N0M0, ER-/PgR- }\end{array}$ & $\begin{array}{l}53 \\
54\end{array}$ & & & \\
\hline $632-8$ & 3450delCAAG & DC, grIII, T1N1M0, ER-/PgR- & 41 & & & \\
\hline $743-6$ & 816delGT & $\begin{array}{l}\text { Atypical medullary cancer, } \\
\text { grIII, T2N2M0, ER-/PgR- }\end{array}$ & 36 & & & \\
\hline $793-4$ & & DC, grII, T1N1M0, ER+/PgR+ & 59 & & & \\
\hline $797-1$ & 1675 delA & DC, grIII, T1NXM0, ER-/PgR- & 49 & & & \\
\hline $837-5$ & & & & Serous, grIII, stIII & 63 & \\
\hline $837-6$ & & $\begin{array}{l}\text { DC, grIII, T1N0M0, ER- } \\
\text { DC, grIII, T1N0M0, ER-/PgR- }\end{array}$ & $\begin{array}{l}69 \\
69\end{array}$ & & & OC 60 \\
\hline $839-1$ & & & & Granulosa cell, stI & 51 & BC 49 \\
\hline $\begin{array}{l}988-1 \\
1048-13\end{array}$ & 3347delAG & DC, grII, T2N0M0, ER+/PgR+ & 39 & Serous, grIII, stIII & 51 & BC 36 \\
\hline $1112-4$ & G3297T & & & Serous, grIII, stIII & 46 & \\
\hline $1353-1$ & 1135 ins A & Lobular, T3N1M0, ER+/PgR+ & 48 & & & \\
\hline 2031-1 & 1675delA & & & Serous, grII, stIII & 53 & \\
\hline
\end{tabular}

\footnotetext{
* Truncated protein by PTT in exon 11, sequence of mutation to be determined.
} 
BRCA1 mutations causing premature translation termination were found in $21(60 \%)$ of the 35 patients (Table 1). Eleven (52\%) out of the 21 mutations corresponded to the two earlier described Norwegian founder mutations, 1675delA $(n=7)$ or 1135insA $(n=4)$. The remaining mutations were dispersed over the large exon 11 and in exon 17.

We have previously estimated that $87 \%$ of the prospectively observed cancers in our material are caused by an inherited predisposition [10]. Assuming that the majority of inherited breastovarian cancer is caused by germline BRCA1 or BRCA2 mutations, we would expect to find approximately $30 \quad(35 \times 0.87)$ carriers of a mutation in either gene. We identified $21(70 \%$ of 30) carriers of a BRCA1 mutation, which may reflect that the presumed remaining 30\% $(\mathrm{n}=9)$ are caused by mutations in BRCA2 or other genes, or by BRCA1 mutations that have escaped detection with our present techniques. Moreover, $11(37 \%)$ of the assumed 30 inherited cancers would have been caused by one of the two BRCA1 founder mutations.

Taking histological type into consideration also, the role of BRCA1 mutations in defining a distinct group of familial breast-ovarian cancer becomes even clearer. Ten (91\%) out of the 11 infiltrating epithelial ovarian cancers were found to carry BRCA1 mutations, as compared to one out of five of the other ovarian cancers $(p=0.004)$. Nine $(82 \%)$ of 11 BRCA1 mutation carriers had grade III ovarian cancers, one had a grade II tumour and one was a borderline ovarian cancer. Moreover, 10 (91\%) out of 11 BRCA1 ovarian tumours were of histological serous type (three were of mixed serous/endometroid type), and one was a poorly differentiated adenocarcinoma not otherwise specified. Ten (83\%) of the 12 breast tumours in BRCA1 mutation carriers (one bilateral) were ductal carcinomas, one had an atypical medullary cancer, and one had a lobular cancer. All the ductal cancers (including the medullary cancer) were grade III tumours, except for one which was poorly fixed and described as at least grade II, and one tumour too small to be evaluated.
Eleven out of $12(92 \%)$ BRCA1 breast tumours examined for estrogen receptor status were receptor negative, versus 4 out of $9(44 \%)$ of the mutation negative $(\mathrm{p}=0.03)$. The single case of intraductal carcinoma in situ in this series belonged to the group of patients in which no BRCA1 mutation was identified.

Median age of breast or ovarian cancer diagnosis was higher for carriers of a founder mutation (49.5 years), as compared to carriers of the other mutations (41 years, $\mathrm{p}=0.005$ ).

This was evident also when distinguishing between the breast ( 48.5 vs. 36 years) and ovarian cancer (51.5 vs. 46 years). The nonBRCA1 cases (which may include BRCA2 mutation carriers), had higher median age at diagnosis (53 years) than BRCA1 mutation carriers.

Excluding the 9 patients with cancers prior to inclusion in the prospective follow-up did not change the results presented above. Applying the same methods for calculations in this group of 26 women, $71 \%$ of presumed inherited cancers carried BRCA1 mutations (compared to $70 \%$ above), and $56 \%$ of the BRCA1 mutations were founder mutations (compared to 52\% above). Patients with founder mutations were still significantly older at diagnosis than women with other mutations $(\mathrm{p}=0.002)$.

\section{DISCUSSION}

We have analysed a group of patients, initially classified as belonging to breast-ovarian cancer families, that have developed cancer prospectively during clinical follow-up. A major issue is whether these families are representative for the population, and whether other selection criteria could have been used to better identify a group of patients for analysis and calculation of mutation frequencies. Based on several years of experience in handling Norwegian breast-ovarian cancer families we believe our inclusion criteria are accurate, but we have no confidence limits to validate our assumptions.

Twenty one $(60 \%)$ of the 35 patients in the present study were identified as BRCA1 mutation 
carriers, and $11(31 \%)$ were found to carry a BRCA1 founder mutation. Based on the observation that $87 \%$ of all cancers in breast cancer kindreds arise in genetically predisposed individuals [10], our results suggest that $70 \%$ (21 of 30 presumed inherited cases) of hereditary breast-ovarian cancer in Norway is caused by BRCA1 alterations. Furthermore, one third $(11 / 30,37 \%)$ of these inherited cases are due to two BRCA1 founder mutations. Owing to the comprehensive approach for mutation detection used in the present study and the fact we detected BRCA1 mutations in 10 out of 11 infiltrating epithelial ovarian cancers, we believe that the fraction of false negative BRCA1 analysis is small. However, we cannot exclude the existence of unknown large rearrangements or mutations in non-coding regions of the gene, and the potential impact a founder effect for such a mutation would have. A more realistic explanation however, would be that the remaining inherited cases are caused by mutations in BRCA2 or other susceptibility genes.

In concordance with our population-based study of ovarian cancer [4], we found that the majority of ovarian cancers in BRCA1 mutation carriers were grade III serous type tumours. Also in agreement with a previous report [9], we found that BRCA1 associated breast tumours were mostly oestrogen receptor negative, grade III ductal carcinomas. Taken together, the histopathological findings suggest that cancers arising in BRCA1 mutation carriers are of an unfavourable prognosis.

We found an older age of disease onset for founder mutation carriers, as compared to other mutation carriers. Unpublished results from retrospective analyses of families with these founder mutations support the observed age at onset in women with founder mutations in this study. In a population-based study, median age of ovarian cancer in women with founder mutations was 51 years [4] which is close to 49 years observed in this study. Women in the present study had cancers detected by screening, and were expected to be slightly younger compared with the population-based series. We conclude that the observed age of disease onset for the founder-mutation carrying women in the present study is representative and not deviating due to fluctuation in small numbers.

It is a logical problem how an early-onset dominantly inherited disease with assumed reduced fitness could become prevalent. The general explanation has been genetic drift, and the Norwegian population has indeed experienced a severe bottleneck some 25 generations back, followed by an expansion and potential genetic drift. Presumably, mutations associated with a lower penetrance and/or later disease onset, would more likely be allowed to survive and expand because they are not affecting women in child-bearing age. If this is true, estimates of BRCA mutation penetrance in populations with strong founder effects may be biased and should not be taken as general guidelines [6,12].

Several mechanisms may lead to reduced penetrance. The examined patients may share a genetic, reproductive or environmental background that modifies the mutation penetrance and risk of early onset of disease $[2,11]$. However, many of the families with the founder mutations were genetically distant from each other and would not be supposed to share non-BRCA1-linked genetic factors, and they lived far apart geographically. A position effect related to which part of the gene was affected by each mutation seems unlikely, because other mutations, found to be associated with earlier disease onset, have been located in the close vicinity of the founder mutations.

The two Norwegian founder mutations are not evenly distributed in the population. The 1675delA mutation seems to have originated on the south-west coast of Norway, and the majority of families with this mutation are still living there. Families with the 1135insA mutation are more frequent in the eastern region of Norway. Thus, the concept of national-based guidelines for disease prevention and follow-up of inherited breast-ovarian cancer may be inappropriate. These should perhaps be governed instead by regional, mutation-specific or even familyspecific experiences of disease phenotype and onset. 
We conclude that a large fraction of familial breast-ovarian cancer in Norway is due to BRCA1 mutations and, in particular, two common founder mutations. Testing for these two mutations is simple and would allow us to identify a large fraction of genetically predisposed women for preventive care.

\section{Acknowledgments}

This work was supported by The Norwegian Cancer Society; Bassøe Shipbrokers, Oslo; Edith Kongshem, Oslo; and the Swedish Cancer Society.

\section{References}

[1] Andersen, T.I., Børresen, A.L. and Møller, P. A common BRCA1 mutation in Norwegian breast and ovarian cancer families. Am. J. Hum. Genet. 59, (1996) 486-487.

[2] Chang-Claude, J., Becher, H., Eby, N., Bastert, G., Wahrendorf, J. and Hamann, U. Modifying effect of reproductive risk factors on the age at onset of breast cancer for German BRCA1 mutation carriers. Cancer Res. Clin. Oncol. 123, (1997) 272-279.

[3] Dørum, A., Møller, P., Kamsteeg, E.J. et al. A BRCA1 founder mutation, identified with haplotype analysis, allowing genotype/phenotype determination and predictive testing. Eur. J. Cancer 33, (1997) 2390-2392.

[4] Dørum, A., Hovig, E., Trope, C., Inganas, M. and Møller, P. 3\% of Norwegian Ovarian Cancers are caused by BRCA1 1675 delA or 1135 insA. Eur. J. Cancer 35, (1999) 779-781.
[5] Dørum, A., Heimdal, K., Løvlsett, K. et al. Prospectively detected cancer in familial breast/ovarian cancer screening. Acta Obst. Gyn. Scand. (in press).

[6] Fodor, F.H., Weston, A., Bleiweiss, I.J., McCurdy, L.D., Walsh, M.E.D., Tartter, T.I., Brower, S.T. and Eng, C.M. Frequency and carrier risk associated with common BRCA1 and BRCA2 mutations in Ashkenazi Jewish breast cancer patients. Am. J. Hum. Genet. 63, (1998) 45-51.

[7] Ford, D., Easton, D.F., Stratton, M. et al. Genetic heterogeneity and penetrance analysis of the BRCA1 and BRCA2 genes in breast cancer families. The Breast Cancer Linkage Consortium. Am. J. Hum. Genet. 62, (1998) 676-689.

[8] Hakansson, S., Johannsson, O., Johansson, U. et al. Moderate frequency of BRCA1 and BRCA2 germ-line mutations in Scandinavian familial breast cancer. Am. J. Hum. Genet. 60, (1997) 1068-1078.

[9] Johannsson, O., Idvall, I., Anderson, C., Borg, Å., Barkardottir, R.B., Egilsson, V. and Olsson, H. Tumour biological features of BRCA1 induced breast and ovarian cancer. Eur. J. Cancer 33, (1997) 362-371.

[10] Møller, P., Mæhle, L., Heimdal, K. et al. Prospective findings in breast cancer kindreds. Annual incidence rates according to age, stage at diagnosis, mean sojourn time, and incidence rates for contralateral cancer. The Breast 7, (1998) 5559.

[11] Phelan, C.M., Rebbeck, T.R., Weber, B.L. et al. Ovarian cancer risk in BRCA1 carriers is modified by the HRAS1 variable number of tandem repeat (VNTR) locus. Nat. Genet. 12, (1996) 309-311.

[12] Thorlacius, S., Struewing, J.P., Hartge, P. et al. Population-based study of risk of breast cancer in carriers of BRCA2 mutation. Lancet 352, (1998) 1337-1339. 


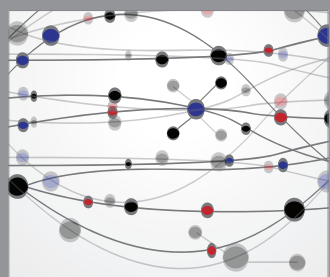

The Scientific World Journal
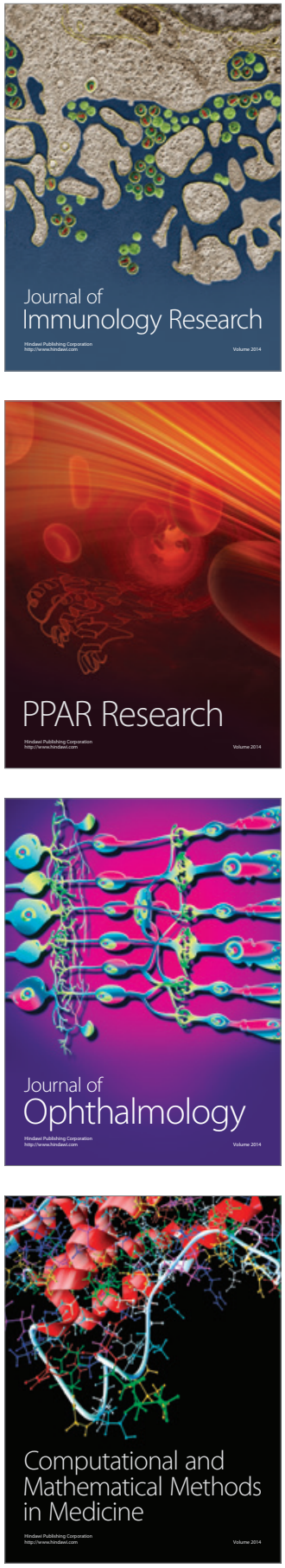

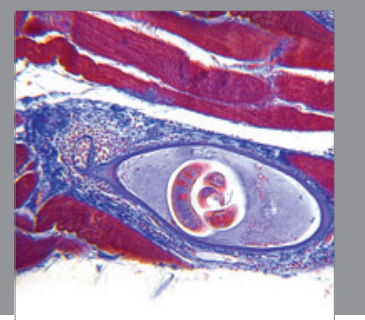

Gastroenterology

Research and Practice
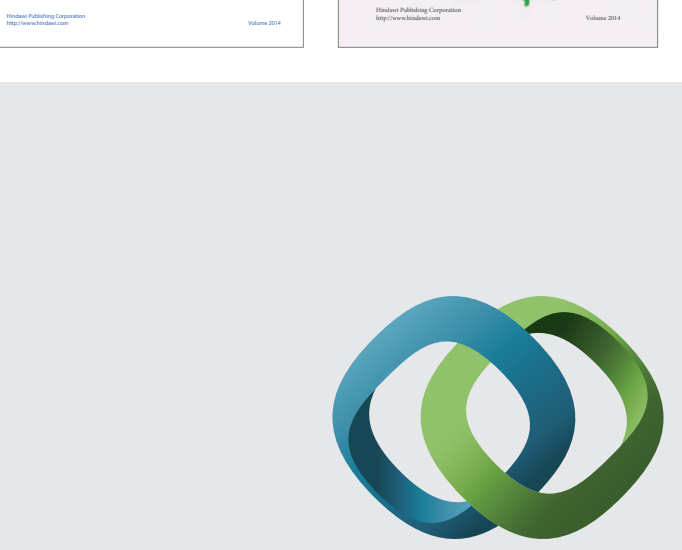

\section{Hindawi}

Submit your manuscripts at

http://www.hindawi.com
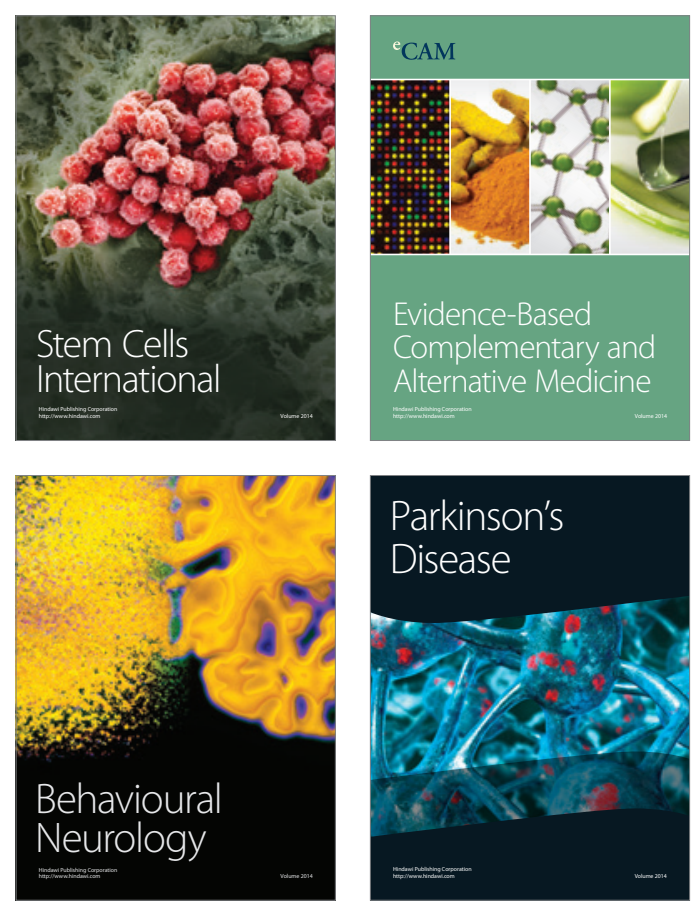

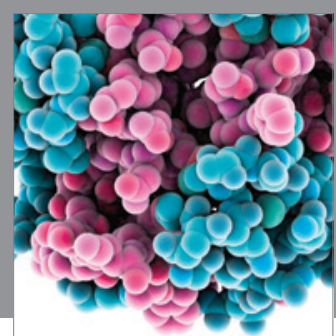

Journal of
Diabetes Research

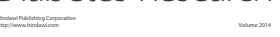

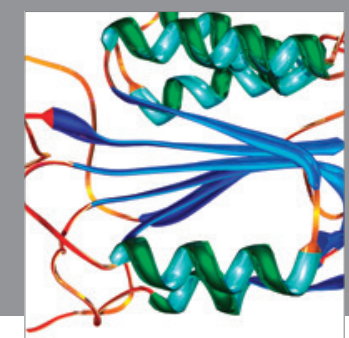

Disease Markers
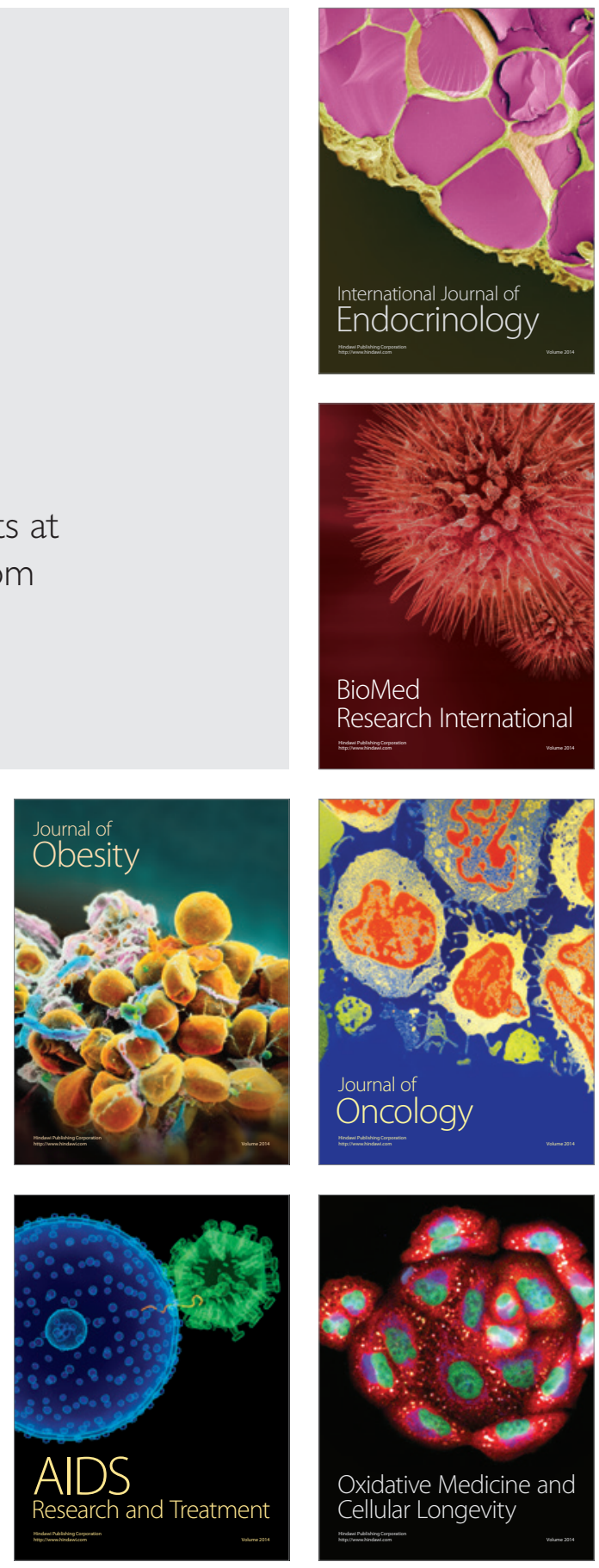